\title{
Finite Element Calculation and Experimental Verification of the Fibers Mechanical Properties in the Reversing Process
}

\author{
Chenchen Han, Wenliang Xue, Longdi Cheng \\ Key Laboratory of Textile Science \& Technology, Ministry of Education, Donghua University, Shanghai 201620, People’s \\ Republic of China
}

\begin{abstract}
Through the three-dimensional solid fiber model built by ANSYS finite element software, it calculated and analyzed the torsional mechanical (stress / strain) performance of cotton, wool, polyester and other fibers. In contrast with the experimental results, it validates the feasibility and effectiveness of ANSYS finite element calculation of the fibers mechanical properties in the reversing process, and provides the basis for experimental studies and theoretical analysis.
\end{abstract}

Keywords-fiber model; ANSYS; finite element analysis; torsion.

\section{INTRODUCTION}

The fiber has a large aspect ratio, a certain flexibility and a flexible elongated body, so the fibers in the spinning process will exhibit translation, rotation and other complex dynamical behaviors, as well as bending, twisting, stretching and other non-linear distortions. The researchers use more experimental means to study torque size, torque stress and other relaxation properties of fibers. The test methods and principles can be basically divided into two categories: wiggler type [1-7] and torque scale type [8-14]. Theoretical researches about dynamical behaviors of fibers in the spinning process should first establish the model can reasonably describe the uniqueness of the fibers. Due to the lack of model which can fully reflect the characteristics of the fibers, the theoretical study of the mechanical properties of fiber torsion is rarely reported.

In this paper, the nonlinear fiber torsional deformation is simulated by finite element ANSYS software and the finite element analysis results with the corresponding experimental results are compared. Three-dimensional solid model, based on the characteristics of the material structure, selects the most appropriate units. Finite element analysis would not be affected by the number of samples, and can reduce the impact of the various random factors in experiments, largely saving resources compared to experimental study.

\section{II.ESTABLISHMENT OF NONLINEAR FINITE ELEMENT MODEL}

\section{A. Definition of unit type}

The fiber uses SOLID164 solid element. SOLID164 is an 8-node solid element, which can be applied to a single point of integration and multi-points of integration solid element algorithm for large deformation. The time of the calculation is shorter [15].

\section{B. Definition of material properties}

The material parameters of specific fiber properties [16] are shown in Table I. Because in the actual spinning process, deformation of the individual fiber is small, which does not involve structural changes between the fiber molecules. This paper uses the linear elastic model to simplify the simulation. Pre tensile stress is $0 \mathrm{KN}$, according to the consideration of an ideal elastic-plastic model, and ultimate tensile strain is taken as 0.01 . The constitutive relation for linear elastic fibers is:

$$
\sigma_{f}=E_{f} \varepsilon_{f}
$$

TABLE I. MATERIAL PARAMETERS

\begin{tabular}{cccc}
\hline Material & $\begin{array}{c}\text { Density } \\
\left(\mathrm{kg} / \mathrm{m}^{3}\right)\end{array}$ & $\begin{array}{c}\text { Poisson } \\
\text { ratio }\end{array}$ & $\begin{array}{c}\text { Elasticity modulus } \\
(\mathrm{Pa})\end{array}$ \\
\hline $\begin{array}{c}\text { Cotton } \\
\text { fiber }\end{array}$ & 1500 & 0.35 & $4.6 \times 10^{11}$ \\
$\begin{array}{c}\text { Wool fiber } \\
\text { Polyester } \\
\text { fiber }\end{array}$ & 1250 & 0.30 & $1.47 \times 10^{11}$ \\
\hline
\end{tabular}

C. Definition of geometric model

In order to compare with experimental results, the geometry parameters of finite element model are shown in Table II.

TABLE II. MODEL GEOMETRY

\begin{tabular}{ccc}
\hline Material & Length $(\mathrm{mm})$ & Radius $(\mathrm{mm})$ \\
\hline Cotton fiber & 28 & $6.20 \times 10^{-3}$ \\
Wool fiber & 100 & $13.25 \times 10^{-3}$ \\
Polyester fiber & 55 & $6.55 \times 10^{-3}$ \\
\hline
\end{tabular}




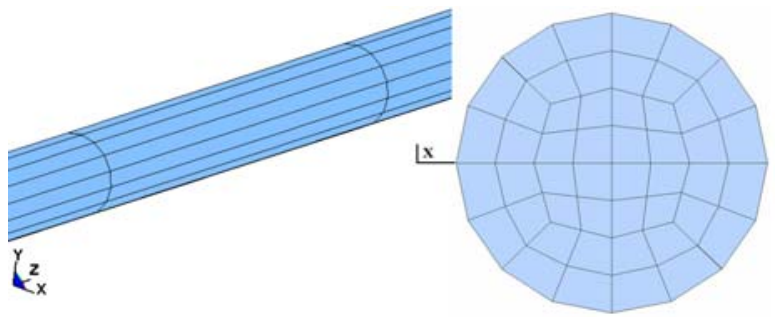

Figure 1. Fiber finite element model

\section{Meshing}

In some cases these irregular units may lead to abnormal end of calculating. Therefore, try to avoid using these irregular units [15], and divide entity grid using regular hexahedron. Set Relevance Center as Fine. Element Size is 0.001. Smoothing is High. Set Transition as Fast. Set Span Angle Center as Fine. Minimum Edge Length is 1e-6m. It is shown in Figure 1, the others are default values.

\section{E. Convergence criterion}

This article uses a convergence criterion based on force and displacement together [15], selecting second norm to control residual stress convergence. Convergence precision can get slightly larger, but not more than 0.05. In this paper, the convergence precision is set to 0.05 , and the number of iterations is set to 50 times.

\section{F. Loading conditions}

In order to apply displacement constraints or loads, plus a common loading surface at each end of the fiber,. Fixed Supports of xyz directions are applied to one common loading surface. Set uniform tangential displacement constraints on the other common loading surface, displacement of which is $720^{\circ}$. Fixed Supports of $\mathrm{z}$ direction is applied to it too, displacement of which is $0 \mathrm{~m}$. The time of load steps is 0.01 second. Load steps are 200. The maximum number of steps is 1000 . In the analysis, fiber length is along the $\mathrm{Z}$-axis direction, and the radial directions is $\mathrm{x}, \mathrm{y}$ axis.

\section{G. Analysis method}

Use the following assumptions in the finite element analysis: (1) it does not consider slippage between fibers. Because the current study about bond-slip relationship between fibers is still not mature enough. The adhesion strength of the contact surface is difficult to obtain; (2) Under force, strain deformation strain of the fibers and yarns satisfy the principle of coordination; (3) The shear capacity of fiber is enough before and after the nonlinear deformation.

Newton - Raphson iterative method can help to modify the stiffness matrix at different start times [15]. Newton Raphson iterative method is based on squared convergence. The speed of convergence is faster, but the computational workload is large, and relatively easy to diverge; Initial stiffness method is based on linear convergence. But it needs more iterations, and the speed of convergence is slow; Modified Newton - Raphson iteration method stays between the two methods. This paper selects modified Newton Raphson iterative method for the nonlinear finite element analysis of fiber mechanical properties in the torsional process.

\section{RESULTS ANALYSIS}

\section{A. Calculation results}

In the twisting process, the strain is gradually reduced from the inner to the outer layer. As the torsional deformation increases, the shear force on the interior of the fiber increases. ANSYS nonlinear finite element analyzes the two single-fiber models, and the computational results are shown in Figure 2. It shows the distribution of deformation. It also can get the strain versus time curve, the stress versus time curve, stress-strain curve of a point on the fiber. By changing different material properties and geometry models, this method can solve the measuring problems of short fiber reversing performance, as well as a variety of filament fibers. According to the article needing, it mainly analyzes and compares the stress-strain curve in the twisting process of different fiber materials.

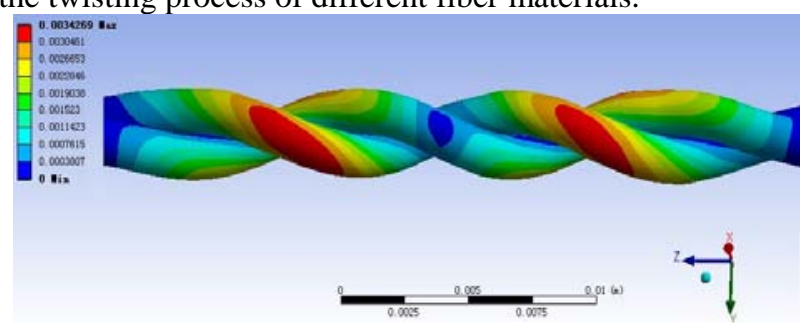

Figure 2. Torsional deformation distribution of the fiber

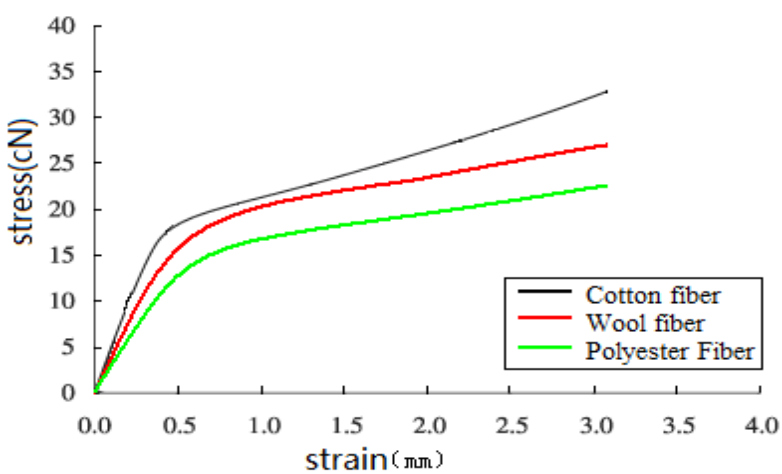

Figure 3. Comparison chart of ANSYS torsional stress and strain values

TABLE III. TORSIONAL PROPERTIES OF THE FIBERS

\begin{tabular}{cccc}
\hline Material & $\begin{array}{c}\text { Torsional } \\
\text { section shape } \\
\text { factor }\end{array}$ & $\begin{array}{c}\text { Ratio shear } \\
\text { modulus(cN/tex) }\end{array}$ & $\begin{array}{c}\text { Relative } \\
\text { torsional } \\
\text { stiffness } \\
\left(\mathrm{cN} \cdot \mathrm{cm}^{2} / \mathrm{tex}^{2}\right)\end{array}$ \\
\hline $\begin{array}{c}\text { Cotton } \\
\text { fiber }\end{array}$ & 0.71 & 161.7 & $7.74 \times 10^{-4}$ \\
$\begin{array}{c}\text { Wool } \\
\text { fiber }\end{array}$ & 0.98 & 83.3 & $6.57 \times 10^{-4}$ \\
$\begin{array}{c}\text { Polyester } \\
\text { Fiber }\end{array}$ & 0.99 & 63.7 & $4.61 \times 10^{-4}$ \\
\hline
\end{tabular}




\section{B. Contradistinction of calculation results}

The finite element calculating results of three different fiber materials at the $\mathrm{Z}=10 \mathrm{~mm}$ are compared in Figure 3 . Fiber torsional deformation generally is divided into two phases: The first phase of deformation under external force is that the bond lengths and bond angles of macromolecular backbone gradually increase, and the secondary bonds of the molecular bonds gradually elongate. The torsional deformation and force of the fiber are proportional. The second phase is that under the external force, a part of the molecular chains in amorphous region extend from the crimped state gradually along the direction of the force. At this time, the segment of the molecule must overcome various remote or proximity second valence bond force of intermolecular or intramolecular, so the fiber torsional process is relatively slow. Actual torsional performance [15] of fiber materials is shown in Table III. The relative torsional stiffness of cotton fibers is the biggest, followed by wool fibers and that of polyester fiber is the least. The trend of torsional deformation and the relative size of the torsional stiffness in the finite element calculating results are realistic.

\section{Contradistinction of experimental results}

Based on KES-YN-1[16] the commercial yarn torque tester, which is a multi-purpose torque, and a cross rotary torque test machine, the torque and cross change position relevant data of silk or hairs can be measured. The length of upland cotton experimental fiber is between 23 and 33mm, and the diameter is $11.0-13.8 u m$. The length of Australia imported wool is $90-110 \mathrm{~mm}$, and the diameter is $23-30 \mathrm{um}$. The length of polyester staple fiber is $51-65 \mathrm{~mm}$, and the diameter is $12.0-14.2 \mathrm{um}$. Temperature test environment is $20^{\circ} \mathrm{C}$, and the relative humidity is $60 \%$.

Comparison of the torque test experimental results and the finite element results are shown in Figure 4 (a), (b), (c). It has a good agreement. The inhomogeneity of fibers, and the error of experiment may led to the deviation between finite element analysis and experimental results. But it is still within the allowed range. In the Figure 4 (a), it can be seen the torsional stress-strain curve of cotton fiber. The data of the experimental results is mostly located at the bottom of the curve of the finite element results. This is mainly because that in the ANSYS finite element analysis of fiber, the geometry is defined as a solid cylinder, while the actual cotton fiber structure has multi chambers. It is thick in the middle, and the tail is slightly thin. It looks like a flat ribbon, with a natural twist, which is called " convolution ". Special structure of cotton fiber makes the difference with the finite element model and because of its natural twist and the cavity structure make the corresponding stress of experimental results generally small in the same strain compared to the finite element results. In the Figure 4 (b), the comparison is in a good agreement. Mainly because that the cross-section of wool fiber is round or oval. And the fiber is divided into scaly layer , cortex layer and medulla layer from outside to inside. The surface roughness makes the differences with the fiber geometry of finite element analysis, resulting in an error, but the error is smaller than the cotton fiber. In the Figure 4 (c), the error of polyester fiber is minimized compared with cotton fiber and wool fiber. Because polyester fiber is produced by melt spinning method. The morphological structure observed in the microscope has a circular cross-section and no special vertical structure. It is closest with solid cylindrical geometry model defined by the finite element analysis. Therefore, the definition of the geometric model has a greater impact on the mechanical properties of fiber torsion. It is recommended that in future, the finite element calculation should try to choose the ideal model that is close to the real shape, in order to reduce errors.

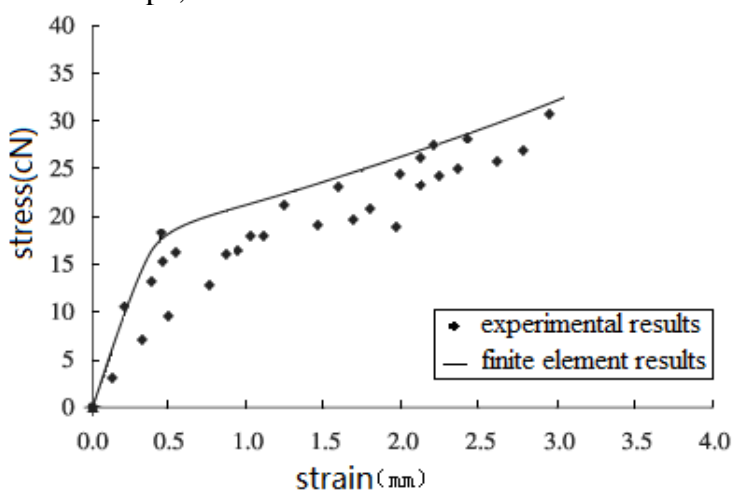

(a) Cotton fiber

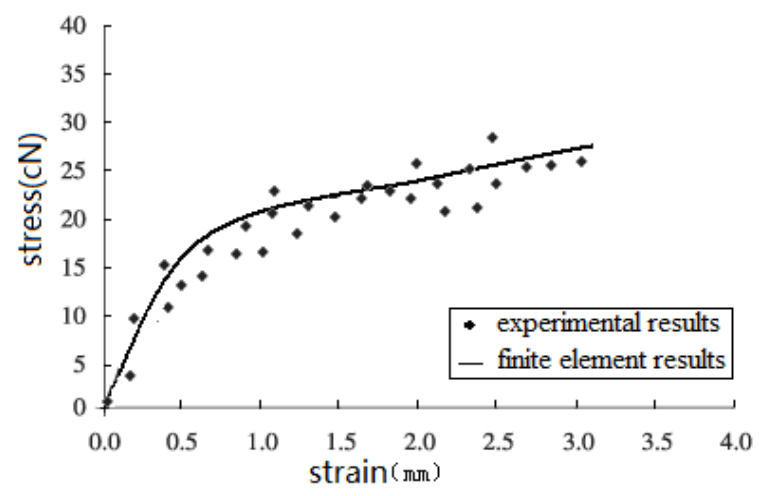

(b) Wool fiber

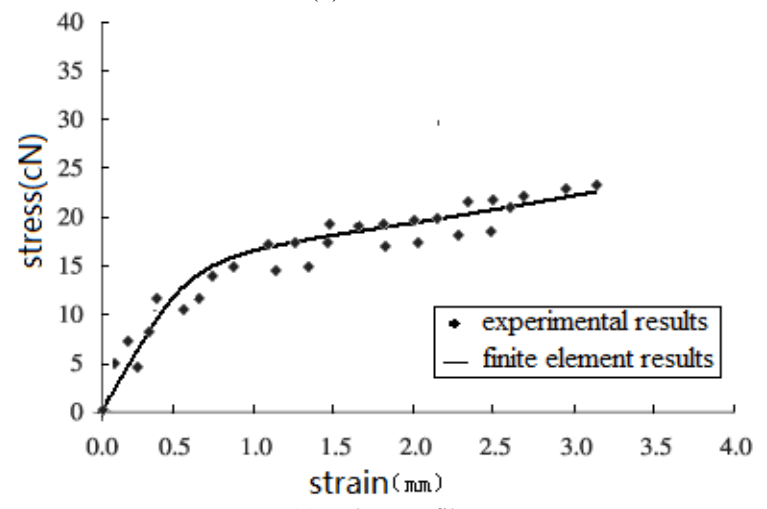

(c) Polyester fiber

Figure 4. Comparison chart of ANSYS values and experimental values 


\section{CONCLUSION}

(1) ANSYS FEM can reasonably define material properties and geometry model to calculate the mechanical properties of different materials and structural fibers in reversing process. ANSYS finite element analyzing fiber torsional deformation is an effective method, providing a basis for experimental research and theoretical analysis, to lay a foundation for further analysis of stress mechanism and deformation of the fibers in the yarn during spinning processing.

(2) According to the comparison of the torque test experimental results and the finite element results: the establishment of a reasonable finite element model, and the use of reasonable solution strategy, can make a good agreement. ANSYS finite element analysis can be an effective alternative of experimental analysis, to some extent, thereby reducing the time and cost required for the experiment.

\section{ACKNOWLEDGMENTS}

This work is supported by Shanghai Natural Science Foundation (No 13ZR1400900); Keygrant Project of Chinese Ministry of Education (No 113027A); and Fundamental Research Funds for the Central University (No 14D110109).

\section{REFERENCES}

[1] Karrholm M, Nordhammer G and Friberg O. Penetration of alkaline solutions into wool fibers determined by changes in the rigidity modulus. Text Res J, 25, pp. 922-929, 1955.

[2] Goodings A C. A method for the measurement of rigidity of fibers immersed in liquids: the torsion double pendulum. Text Res $J$, 38, pp. 123-129, 1968.

[3] Owen J D. The application of Searle's single and double pendulum methods to single fiber rigidity measurements. $J$ Text Inst, 56, pp. 329-339, 1965.

[4] Meredith R. The torsional rigidity of textile fibers. J Text Inst, 45, pp. 489-503, 1954.

[5] MeredithR. The determination of tensile properties and torsional rigidity of fibers. $J$ Text Inst, 38, pp. 17-19, 1947.

[6] Guthrie J C, Morton D H and Oliver P H. An investigation into bending and torsional rigidities of some fibers. $J$ Text Inst, 45, pp. 912-929, 1954.

[7] Zurek W, Durska I. The torsional rigidity of blend yarns. Text Res J, 50, pp. 555-567, 1980.

[8] Peirce F T. The plasticity of cotton and other materials. $J$ Text Inst, 14, pp. 390-413, 1923.

[9] Morton W E, Permanyer F. The measurement of torsional relaxation in wet and dry fibers. $J$ Text Inst, 38, pp. 54-59, 1947.

[10] Mitchell T W, Feughelman M. The torsional properties of single wool fibers, PartI: torque-twist relationships and torsional relaxation in wet and dry fibers. Text Res $J, 30$, pp. 662-667, 1960.

[11] Postle R, Burton P, Chaikin M. The torque in twisted singles yarns. $J$ Text Inst, 55, pp. 461-488, 1964.

[12] Skelton J. The measurement of the torsional elastic recovery of filaments. J Text Inst, 56, pp. 443-453, 1965.

[13] Okabayshi M, Yamzaki C. Measurement of torsional rigidity of fibers. Text Res J, 46, p. 429-433, 1976.

[14] Bennett J M, Postle R. A study of yarn torque and its dependence on the distribution of fiber tensile stress in the yarn, Part II: experimental. J Text Inst, 70, pp. 133-141, 1979.

[15] Weidong Yu. Textile Materials Science (China Textile Press, Beijing 2006).

[16] Kato Tech Co. LTD. Manual for KES-YN-1 Yarn Torsion and Intersecting Torque Tester. 